\title{
Cerebral Palsy—More Than Just Spasticity
}

\author{
Pawan Kumar $^{1} \cdot$ Naveen Sankhyan ${ }^{1}$ (i)
}

Received: 14 December 2021 / Accepted: 22 December 2021 /Published online: 18 January 2022

(c) Dr. K C Chaudhuri Foundation 2021

Children with cerebral palsy have poor motor selection, and consequently, excessive inappropriate motor activity. This results in movement disorders, the most common being dystonia. Pathophysiologically, dystonia results from a lack of selective motor control, excessive synaptic plasticity, and spread of stimuli due to lack of inhibition. The consequent multiple simultaneous muscle activation during a task rather than those most appropriate for the task results in impairment [1]. The complex hyperkinetic movement disorder in cerebral palsy is often referred to as dyskinesia. Traditionally, cerebral palsy has been thought to be synonymous with spasticity, and movement disorders in cerebral palsy have received less attention. It is thus not surprising that reports from registries have possibly under-reported the presence of movement disorders [2]. More recent work and use of standard measuring scales have improved the detection of movement disorders, and in quantifying severity [3]. The work by Suresh et al. in this issue of the Journal has brought to focus the importance of assessing movement disorders in cerebral palsy [4]. The authors highlight that movement disorders may be under-recognized among those children in whom neurological examination tends to be dominated by other motor abnormalities [4]. It is also noteworthy that causes of dyskinetic cerebral palsy like hyperbilirubinemia continue to affect resource-limited nations like India [5].

However, there is more to identification and quantification of movement disorders in cerebral palsy. Firstly, the importance of finding a movement disorder may guide the need for further diagnostic workup. Several well-described conditions such as the dopa-responsive dystonias, aromatic 1-amino acid decarboxylase $(A A D C)$ deficiency, as well as glucose transporter (GLUT1; also known as SLC2A1) deficiency will have the phenotype of cerebral palsy with movement disorder. Not only this, whole exome sequencing is

Naveen Sankhyan

drnsankhyan@yahoo.co.in

1 Department of Pediatrics, APC, Postgraduate Institute of Medical Education and Research, Chandigarh 160012, India expanding the list of genetically inherited disorders as cause of dyskinetic cerebral palsy [6].

Secondly, the study of movement disorders requires specific tools to clinically evaluate the presence and severity of movement disorders in children. Tools that specifically measure dyskinesia in children with cerebral palsy are the Barry-Albright Dystonia Scale (BADS) and the Dyskinesia Impairment Scale (DIS). The DIS measures both dystonia and choreoathetosis, while BADS measures dystonia. The BADS is the preferred choice for dystonia in clinical settings based on its clinical utility. One needs to remember that these scales do not measure how dyskinesia impacts activities, participation, and quality of life of individuals with cerebral palsy. Additionally, applying these scales at one time ignores the day-to-day fluctuations in movement disorders that can arise due to stress, anxiety, pain, and fatigue or other factors [7]. In this context, the study of functional impairments in the setting of cerebral palsy by Suresh et al. is noteworthy [4]. Another aspect is the change in the type and severity of the movement disorder with age. A study with younger children [4] is not directly comparable with one which has older children [3].

Thirdly, movement disorders impact the function of a child with cerebral palsy in many ways. While studying the impact of movement disorders in children with cerebral palsy it is important to go beyond the obvious motor impairments and include assessment of speech, eating, and drinking [8]. Additionally, it has also been reported that a high proportion of children with cerebral palsy and dyskinesia experienced chronic pain. The physical and emotional impacts of living with chronic pain and dyskinesia existed along a spectrum. It has been suggested that affected children may benefit from targeted chronic pain education and management within biopsychosocial models $[9,10]$.

The ultimate goal of study of movement disorders in cerebral palsy is to mitigate its effects. One suggested strategy is managing dystonia at a younger age followed by choreoathetosis at a later stage [11]. However, use of drugs to control movement disorders is hampered by lack of good quality trials in children. Lack of evidence is partially due to the inconsistency of classifications and outcome measures used. It has been emphasized that outcome measures should be 
selected within the framework of the International Classification of Functioning, Disability and Health, in future clinical trials [12]. Not only the drugs, but also the use of interventions like deep-brain stimulation requires evidence from large prospective studies with long-term follow-up using suitable motor and disability evaluation tools. The impact of interventions on neuropsychological and neuropsychiatric aspects, as well as quality of life, should also be better evaluated [13]. The optimum timing of interventions such as deep-brain stimulation is also a subject of research that needs careful study. If successful at an early age, it offers an option to reduce maladaptive neuroplasticity and prevent skeletal deformities [13].

The study by Suresh et al., has brought forth several important aspects of movement disorders in cerebral palsy. It is important to conduct further research in this field to guide management of affected children and improve their participation in the society.

\section{Declarations}

\section{Conflict of Interest None.}

\section{References}

1. Quartarone A, Rizzo V, Morgante F. Clinical features of dystonia: a pathophysiological revisitation. Curr Opin Neurol. 2008;21:484-90.

2. Howard J, Soo B, Graham HK, et al. Cerebral palsy in Victoria: motor types, topography, and gross motor function. J Paediatr Child Health. 2005;41:479-83.
3. Rice J, Skuza P, Baker F, Russo R, Fehlings D. Identification and measurement of dystonia in cerebral palsy. Dev Med Child Neurol. 2017;59:1249-55.

4. Suresh N, Garg D, Pandey S, et al. Spectrum of movement disorders and correlation with functional status in children with cerebral palsy. Indian J Pediatr. 2021. https://doi.org/10.1007/ s12098-021-03785-7.

5. Saini AG, Sankhyan N, Malhi P, Ahuja C, Khandelwal N, Singhi P. Hyperbilirubinemia and asphyxia in children with dyskinetic cerebral palsy. Pediatr Neurol. 2021;120:80-5.

6. Dzinovic I, Śkorvánek M, Pavelekova P, et al. Variant recurrence confirms the existence of a FBXO31-related spastic-dystonic cerebral palsy syndrome. Ann Clin Transl Neurol. 2021;8:951-5.

7. Harvey A. Challenges and advancements in measuring dyskinesia in cerebral palsy. Dev Med Child Neurol. 2020;62:411.

8. Monbaliu E, de Cock P, Ortibus E, Heyrman L, Klingels K, Feys H. Clinical patterns of dystonia and choreoathetosis in participants with dyskinetic cerebral palsy. Dev Med Child Neurol. 2016;58:138-44.

9. McKinnon CT, Morgan PE, Antolovich GC, Clancy CH, Fahey MC, Harvey AR. Pain in children with dyskinetic and mixed dyskinetic/spastic cerebral palsy. Dev Med Child Neurol. 2020;62:1294-301.

10. McKinnon CT, White JH, Morgan PE, et al. The lived experience of chronic pain and dyskinesia in children and adolescents with cerebral palsy. BMC Pediatr. 2020;20:125.

11. Monbaliu E, De La Peña MG, Ortibus E, Molenaers G, Deklerck $\mathrm{J}$, Feys H. Functional outcomes in children and young people with dyskinetic cerebral palsy. Dev Med Child Neurol. 2017;59:634-40.

12. Masson R, Pagliano E, Baranello G. Efficacy of oral pharmacological treatments in dyskinetic cerebral palsy: a systematic review. Dev Med Child Neurol. 2017;59:1237-48.

13. Meoni S, Moro E. Neuromodulation for children with cerebral palsy. Dev Med Child Neurol. 2020;62:6.

Publisher's Note Springer Nature remains neutral with regard to jurisdictional claims in published maps and institutional affiliations. 